\title{
Video Article \\ Characterization of Sickling During Controlled Automated Deoxygenation with Oxygen Gradient Ektacytometry
}

\author{
Minke A.E. Rab ${ }^{1,2}$, Brigitte A. van Oirschot ${ }^{1}$, Jennifer Bos ${ }^{1}$, Celeste K. Kanne ${ }^{3}$, Vivien A. Sheehan ${ }^{3}$, Eduard J. van Beers ${ }^{2}$, Richard van Wijk ${ }^{1}$ \\ ${ }^{1}$ Laboratory of Clinical Chemistry and Hematology, University Medical Center Utrecht, Utrecht University \\ ${ }^{2}$ Van Creveldkliniek, University Medical Center Utrecht, Utrecht University \\ ${ }^{3}$ Department of Pediatrics, Division of Hematology/Oncology, Baylor College of Medicine
}

Correspondence to: Minke A.E. Rab at M.A.E.Rab@umcutrecht.nl

URL: https://www.jove.com/video/60213

DOI: doi:10.3791/60213

Keywords: Medicine, Issue 153, sickling, RBC deformability, deoxygenation, ektacytometry, sickle cell disease, diffraction pattern, hemoglobin

Date Published: $11 / 5 / 2019$

Citation: Rab, M.A., van Oirschot, B.A., Bos, J., Kanne, C.K., Sheehan, V.A., van Beers, E.J., van Wijk, R. Characterization of Sickling During Controlled Automated Deoxygenation with Oxygen Gradient Ektacytometry. J. Vis. Exp. (153), e60213, doi:10.3791/60213 (2019).

\section{Abstract}

In sickle cell disease (SCD), a single point mutation in the gene coding for beta-globin causes the production of abnormal hemoglobin $\mathrm{S}$ (HbS). When deoxygenated, $\mathrm{HbS}$ can polymerize, forming rigid rods of hemoglobin, resulting in the sickling of red blood cells (RBCs). These sickled RBCs have significantly reduced deformability, causing vaso-occlusion, which leads to numerous SCD-related clinical complications, including pain, stroke, and organ damage. RBC deformability is also reduced by RBC dehydration, resulting in dense red blood cells that are more likely to sickle. To date, there is not a single widely available, rapid, and reproducible laboratory assay capable of predicting the disease severity or directly monitoring the treatment effects for novel, non-fetal hemoglobin inducing therapies. In this study, we describe a protocol to measure RBC deformability as a function of $\mathrm{pO}_{2}$ that allows for the quantitation of sickling behavior in SCD patients. Oxygen gradient ektacytometry measures $\mathrm{RBC}$ deformability, expressed as the elongation index (EI), as a function of $\mathrm{pO}_{2}$. $\mathrm{RBCs}$ are exposed to a fixed shear stress of $30 \mathrm{~Pa}$ during one round of deoxygenation and reoxygenation. Six readout parameters are produced. Of these, the point of sickling (PoS), defined as the $\mathrm{pO}_{2}$ at which maximum $\mathrm{EI}\left(\mathrm{El}_{\max }\right)$ shows a $5 \%$ decrease, and minimum El during deoxygenation $\left(\mathrm{EI}_{\min }\right)$ are the most informative, reflecting an individual patient's $\mathrm{pO}_{2}$ at which sickling starts and the minimal deformability of a patient's red blood cells, respectively. PoS is associated with an individual patient's hemoglobin affinity for oxygen, whereas $\mathrm{EI}_{\min }$ shows a strong correlation with fetal hemoglobin levels. We conclude that oxygen gradient ektacytometry is a promising technique to monitor the treatment of patients with SCD, as a biomarker for anti-sickling agents in clinical and preclinical trials, and an important tool to study sickling behavior of RBCs from individuals with SCD and sickle cell traits.

\section{Video Link}

The video component of this article can be found at https://www.jove.com/video/60213/

\section{Introduction}

In SCD, a single point mutation results in the production of $\mathrm{HbS}$, which can polymerize upon deoxygenation. HbS polymerization causes sickling of RBCs and reduces RBC deformability. The combination of RBC sickling and RBC adherence to the endothelium leads to various SCD complications, including vaso-occlusive crises (VOC), stroke, organ damage, and chronic hemolytic anemia. Even at normoxic conditions, RBC deformability is compromised in patients with SCD. Deformability is further decreased at low oxygen concentrations. Key players that determine deformability at normoxia are dense cells, irreversibly sickled cells (ISC), and dehydrated cells, all of which have a decreased surface-to-volume ratio $^{1,2,3}$.

Ektacytometry is an established method to measure RBC deformability, widely used for the diagnosis of hereditary hemolytic anemias, particularly membranopathies ${ }^{4}$. It can also be used to study hemorheology $y^{5,6,7,8,9}$. Osmotic gradient ektacytometry, in which RBC deformability is measured during a continuous change in osmolality, has been used to study SCD for over a decade ${ }^{10,11}$. The percentage of fetal hemoglobin $(\mathrm{HbF})$ is one of the strongest inhibitors of $\mathrm{HbS}$ polymerization because neither $\mathrm{HbF}$ nor its mixed hybrid tetramer (\#2 $\beta S \mathrm{~S}$ ) can enter the deoxyHbS polymer phase ${ }^{12}$. Recent studies suggest that increasing HbF levels in SCD patients leads to a better surface-to-volume ratio, thereby improving the hydration state and thus the deformability in nontransfused patients ${ }^{11}$.

RBC deformability has been studied in the past as a biomarker for SCD complications, but with conflicting results. In studies performed crosssectionally and at a steady state, individuals with higher levels of RBC deformability were found to have a higher incidence of osteonecrosis and more pain crises ${ }^{13,14,15}$. In contrast to these findings, when compared to the steady state values during an acute VOC, RBC deformability was decreased in longitudinal studies within the same individuals ${ }^{16}$. This discrepancy may be the result of studying RBC deformability under different conditions (i.e., during the steady state versus VOC). The percentage of sickled cells is high at the start of a VOC and the cells are rapidly destroyed as the crisis progresses, which may explain the difference between the steady state cross-sectional incidence data and longitudinal data obtained during the VOC. However, other factors, such as adherence of RBC subpopulations to the endothelial surface, may also be important in the occurrence of VOC. In SCD, it is more clinically relevant to measure the deformability during the deoxygenation, because vasoocclusion typically occurs in the hypoxic postcapillary venules and not in the less hypoxic microcapillary network ${ }^{17}$. Additionally, the presence of 
ISCs may alter the ability of an ektacytometer to measure the deformability at normoxia. Distortion of the diffraction pattern is caused by ISCs, which results from the non-alignment during the flow ${ }^{1,2,3}$.

Alternative approaches to study the pathophysiology of VOC include measurements of RBC adherence to an artificial surface ${ }^{18}$, single cell electrical impedance microflow cytometry ${ }^{19}$, microfluidic-based models combining quantitative measurements of the cell sickling and unsickling with single cell rheology ${ }^{20}$, and laser-induced polymerization ${ }^{21}$. Although promising, these techniques are costly, labor intensive, and require extensive operator training. In addition, the assays that are morphology-based lack the ability to study cellular behavior, such as deformability, as a function of an oxygen gradient.

In this study, we describe a rapid and reproducible functional assay performed with an ektacytometer. This is a next generation ektacytometry measurement that measures the different qualitative aspects of RBC deformability expressed as the EI during deoxygenation (1,300 s) and swift reoxygenation (280 s). These time intervals allow for $\mathrm{HbS}$ polymer formation, and thereby the occurrence of morphological changes and then recovery. Deoxygenation occurs by introducing nitrogen gas, which slowly decreases the oxygen tension in the blood sample in the gap between the bob and cup of the ektacytometer. RBC deformability is continuously measured while oxygen tension is measured every $20 \mathrm{~s}$ by means of a small $\mathrm{O}_{2}$-spot present in the wall of the cup. During the test, approximately $80 \mathrm{pO}_{2}$ measurements are coupled to the El measured at that moment. The oxygen pressure drops below $20 \mathrm{mmHg}$ during the deoxygenation, and reoxygenation is facilitated by the passive diffusion of ambient air. The experimental setup of the ektacytometer and oxygen gradient ektacytometry module is described in Figure 1 and Figure 2. The principle of ektacytometry is based on RBC-induced scattering of light from a laser beam. This results in an elliptical diffraction pattern when shear stress is applied at the same time (Figure 1).

\section{Protocol}

All procedures were approved by the ethical committee of the University Medical Center Utrecht (UMCU) and in accordance to the Declaration of Helsinki. Patients enrolled at the Texas Children's Hematology Center (TCHC) were approved by the local IRB and in accordance with the Declaration of Helsinki.

\section{General considerations}

1. Begin by performing a test measurement to warm up the bob and cup. Ensure that the temperature of the bob and cup is $37^{\circ} \mathrm{C}$. This is important for good reproducibility.

2. Ensure that the viscous polyvinylpyrrolidone (PVP) solution falls within the strict limits for osmolarity $(282-286 \mathrm{mOsm} / \mathrm{kg})$, $\mathrm{pH}(7.35-7.45)$ and viscosity $(27.5-32.5 \mathrm{MPa})$ at room temperature $\left(22^{\circ} \mathrm{C}\right)$.

NOTE: The PVP must be used at room temperature. If stored at a lower temperature, make sure it has warmed up to room temperature prior to taking any measurements.

\section{Start-up of the ektacytometer}

1. Switch on the computer and the ektacytometer from the back. Start the software program (Table of Materials) on the computer.

2. Make sure the nitrogen is available to deoxygenate the sample by opening the nitrogen cylinder.

3. Lower the bob in the cup and make sure the cup can turn freely. Clean the cup on the inside and outside with a soft cloth and distilled water because debris can hamper the El measurements.

4. When the software program is running, check for the following message on the screen: "Make sure the gas valve is open" and click OK.

5. Ensure that the ektacytometer starts the $\mathrm{pO}_{2}$ self-check process that will appear on the screen. Select Start (enter). If it fails, rerun the selfcheck by clicking Hardware check $\left|\mathrm{pO}_{2}\right|$ Self check.

NOTE: If the self-check fails again, consider replacing the $\mathrm{O}_{2}$-spot. The $\mathrm{O}_{2}$-spot is replaced by gently pushing the spot out from the inside of the cup with a fingertip. A new spot is placed by gently pushing the spot from the outside into the cup.

6. Choose $\mathbf{p O}_{2}$ scan from the different tests listed on the left. Choose Settings at the right of the screen and ensure they are set as per the parameters listed in Table 1. Keep the same settings for every measurement.

7. In order to save these settings, press OK|OK.

NOTE: Preferred settings are listed in Table 1 but can be adjusted according to the user preferences and investigational purposes. For example, to study the sickling behavior more extensively, deoxygenation speed and duration can be altered.

\section{Sample collection and preparation}

NOTE: For the validation of the technique, ethylenediamine tetraacetic acid (EDTA)-treated blood from 38 SCD patients and 5 healthy controls included at the University Medical Center Utrecht or Texas Children's Hematology Center, in different clinical studies (Netherlands Trial Registry [NTR] identifier, NTR 6779 and NTR 6462), as well as anonymized leftover blood samples from patients who visited the outpatient clinic or were hospitalized were used.

1. Collect blood samples by venipuncture (a minimum of $300 \mu \mathrm{L} / \mathrm{sample}$ ) in a tube containing EDTA. Make sure the blood has been stored for at least 30 min at $4{ }^{\circ} \mathrm{C}$, but no longer than $24 \mathrm{~h}$.

NOTE: Citrate phosphate dextrose adenine (CPDA) or heparin can also be used, but the influence of these reagents on the sample preservation with respect to the oxygen gradient ektacytometry is not well-known.

2. Mix the sample gently by inversion to homogenize. Do not shake the sample. Let the sample warm up to room temperature on a roller bench before the measurement.

NOTE: A sample tube $(9-10 \mathrm{~mL})$ that is stored for more than $1 \mathrm{~h}$ at $4{ }^{\circ} \mathrm{C}$ must warm up for $15 \mathrm{~min}$. When stored for less than $1 \mathrm{~h}$ at $4{ }^{\circ} \mathrm{C}$, it must warm up for $10 \mathrm{~min}$. A sample tube (2-6 mL) that is stored for more than $1 \mathrm{~h}$ at $4{ }^{\circ} \mathrm{C}$ must warm up for 10 min. When stored for less than $1 \mathrm{~h}$ at $4{ }^{\circ} \mathrm{C}$, it must warm up for $5 \mathrm{~min}$. 
3. Measure the complete blood count on a hematology analyzer. To do so, take 20-200 $\mu \mathrm{L}$ of whole blood in a tube containing EDTA. Place the aspiration needle in the tube and press on the button behind the needle of the hematology analyzer to start the measurement. NOTE: In the complete blood count, the RBC number is measured, which is an important factor for standardizing the oxygen gradient ektacytometry measurements. RBC count is calculated from forward and sideward scatter by flow cytometry. Normal RBC count in healthy controls is $3.7-5.0 \times 10^{12} / \mathrm{L}$ for females and $4.2-5.5 \times 10^{12} / \mathrm{L}$ for males. RBC count in patients with SCD is generally decreased. Some hematology analyzers will also measure percent dense red blood cells (\% DRBC) which can be of additional value in the interpretation of individual oxygen gradient ektacytometry curves.

4. Standardize the whole blood sample to an RBC count of $200 \times 10^{6} \mathrm{RBCs}$ in $5 \mathrm{~mL}$ PVP $\left(200 \times 10^{6} \mathrm{RBC}\right.$ /vial $)$ by adjusting the volume of sample that will be added. If the total RBC count is less than $200 \times 10^{6}$, the diffraction pattern and El will be affected.

1. Use the equation below to perform the counting. $4.0 / \times x\left(\times 10^{12} / \mathrm{L}\right) \times 50=$ yy $\mu \mathrm{L}$ whole blood/vial PVP

where $x x$ is the calculated RBC count obtained from step 3.3 and yy is the amount of whole blood that is required for the actual measurement. Depending on the grade of anemia and other factors influencing RBC counts, the amount of whole blood required is 40 $90 \mu \mathrm{L}$.

\section{Oxygen gradient ektacytometry measurement}

1. Pipette the calculated sample volume ( $y y \mu \mathrm{L}$ of blood) into PVP to obtain a total volume of $5 \mathrm{~mL}$. Prewet the tip by gently resuspending the blood 3x. Use a pipette tip with a wide opening to avoid additional stress on the RBCs. Gently mix the sample manually by inversion until it is homogeneous.

NOTE: Open the PVP vial for as short a time as possible to avoid air contact.

2. Slowly draw $2.0 \mathrm{~mL}$ of the blood/PVP mixture into a $3 \mathrm{~mL}$ syringe without the needle. Push the plunger to remove any visible air bubbles and excessive sample solution until $1.5-1.8 \mathrm{~mL}$ is left in the syringe (depending on the cup volume).

3. Inject the total sample volume slowly and evenly in the bob through the connector. Make sure the level of the sample is above the oxygen sensor (pink spot) and above the small suction hole. Do not leave any sample solution in the syringe.

4. Click New and fill in the sample identifier, remarks, date of donation, and viscosity of PVP. Click OK |Aspirate. After $60 \mathrm{~s}$, the cup will rotate and aspirate the sample for $15 \mathrm{~s}$. Click OK when the rotation stops. Close the machine lid. Click Continue | Start now, as oxygen gradient ektacytometry is done with a fixed gain. The measurement will take about $28 \mathrm{~min}$.

5. After the measurement, print the report that shows the curve and parameters that are automatically calculated by the software. Ensure that the raw data is automatically stored in the designated folder in Settings. Maximum El $\left(\mathrm{El}_{\max }\right)$, minimum $\mathrm{EI}\left(\mathrm{EI}_{\min }\right), \mathrm{pO} \mathrm{O}_{2} @ 95 \% \mathrm{El}(\mathrm{PoS})$, and area (area under the curve) are automatically calculated and added to the printed report and raw data.

6. Manually obtain $\Delta \mathrm{El}$ by calculating the difference between $\mathrm{El}_{\max }$ and $\mathrm{El}_{\min }$. Calculate the percentage recovery by taking the difference in mean El before deoxygenation $\left(\mathrm{pO}_{2} 100-120 \mathrm{mmHg}\right)$ and mean El values during reoxygenation at $100-120 \mathrm{mmHg}$.

\section{Cleaning of the ektacytometer}

1. Remove the sample syringe and replace it with a syringe filled with distilled water or deionized water.

2. Press Clean, slowly flushing the connector during rinsing. Make sure to flush in both directions.

3. Remove the syringe and lift the bob. Dry the bob, cup, and connector thoroughly with a soft cloth.

4. Use a large syringe $(10-50 \mathrm{~mL})$ to flush the connector in order to remove any water remaining in the tubes and bob. Block the lower inlet/ outlet of the bob to get back pressure in the tubes, thereby removing remaining water.

5. Lower the bob in the cup. The machine is now ready for the next measurement.

\section{Shutdown of the machine}

1. Ensure the machine is properly rinsed after the last measurement, as described above. Ensure the proper tubes relate to the cleaning solution.

2. Close the software, press Close, and press Start to start end-of-day cleaning program.

3. After completing the whole cleaning program, remove the syringe and lift the bob. Flush the connector with a big syringe.

4. Empty the waste bottle and dry the bob and cup with a soft cloth. Flush the connector in order to remove the water remaining in the tubes and bob. Block the lower inlet/outlet of the bob to get back pressure in the tubes, thereby removing any remaining water.

5. Close the lid of the machine. Close the nitrogen cylinder. Turn off the computer and the machine.

\section{Representative Results}

Oxygen gradient ektacytometry can be used to characterize sickling behavior in patients with SCD. In this study, blood samples from a total of 38 SCD patients and five healthy controls were included. In healthy controls, the diffraction pattern is circular at rest and elliptical at higher shear stress $^{4}$. From the elliptical diffraction pattern, the elongation index (EI) is calculated based on the height and width of the diffraction pattern. In oxygen gradient ektacytometry, slow and continuous deoxygenation of the sample by nitrogen gas is followed by swift reoxygenation by ambient air. Under these conditions, RBC sickling can be observed under deoxygenation. This will cause a distortion of the diffraction pattern because sickled red cells will not align properly under the applied shear stress. Hence, they appear to be less deformable as opposed to healthy RBCs (Figure 2).

Figure 3A shows how sickle RBCs change in shape upon deoxygenation, which mimicked conditions during oxygen gradient ektacytometry, whereas control sickle RBCs without deoxygenation show no change in shape. This process results in distortion of the diffraction pattern during 
oxygen gradient ektacytometry, and thus in a decrease in EI. Figure 3B shows the different diffraction patterns from which different parameters are generated.

A representative curve obtained by the ektacytometer is shown in Figure 3C. Six parameters reflect different characteristics of sickling behavior of RBCs: $\mathrm{El}_{\max }$ is the maximum El at the start of the measurement before deoxygenation. This parameter represents the baseline position and reflects the overall deformability of the total $\mathrm{RBC}$ population at ambient air. $\mathrm{EI}_{\min }$ is the minimum El, which represents minimal deformability after deoxygenation. This parameter reflects changes in the shape and orientation of (sickle) RBCs upon deoxygenation. $\triangle E l$ is the difference between $\mathrm{EI}_{\max }$ and $\mathrm{EI}_{\min }$, which indicates how many cells can sickle during one round of deoxygenation. $5 \%$ Point of Sickling $\left(\mathrm{PoS}_{5 \%}\right)$ is the pO ${ }_{2}$ $(\mathrm{mmHg})$ at which a $5 \%$ decrease of $\mathrm{EI}_{\max }$ during deoxygenation is measured. This represents the oxygen tension where the sickling process starts. Area reflects the area under the curve, which is determined by an integral calculation of $\mathrm{El}$ and $\mathrm{pO}_{2}$ measurements between $100 \mathrm{mmHg}$ and $\mathrm{pO}_{2} \mathrm{~min}(\mathrm{mmHg})$. This is the result of previously described parameters $\mathrm{EI}_{\max }, \mathrm{EI}_{\min }$, and PoS. Recovery represents the difference of $\mathrm{El}$ during the final part of reoxygenation compared to El at baseline. Both El values are measured at a pO $\mathrm{p}_{2}$ of $100-120 \mathrm{mmHg}$. This parameter reflects the capacity of RBCs that sickle during deoxygenation to reverse sickling during reoxygenation ${ }^{22}$. Parameters from duplicate measurements generally had a coefficient of variation (CV) $<5 \%$ (median $1.83 \%$ ). In case a CV $>5 \%$ was obtained, a third measurement was performed. The parameters $\mathrm{EI}_{\max }$ and Recovery are the most reproducible with median CVs $<1 \%$.

Representative curves of RBCs of healthy controls, patients with $\mathrm{HbS}$ traits (heterozygous $\mathrm{HbS}$ ), and a homozygous SCD patient are shown in Figure 4A. The representative curve of the HbSC patient shows a lower recovery, which might indicate a different sickling process (Figure 4B). The representative curves of HbSS patients treated with hydroxyurea (HU) and transfusion are shown in Figure 4C and Figure 4D. Clearly, there is a big difference between the representative curves of HS traits (HbAS cells) and RBCs of HbSS patients treated with transfusion (consisting of a mixture of homozygous sickle (HbSS) and homozygous normal (HbAA) cells, Figure 4A,D). The clear differences in the curves of the untreated SCD patient and the HU and transfusion-treated patients highlights the usefulness of this assay (Figure 4C,D). Levels of HbF and $\mathrm{HbS}$ correlated significantly with $\mathrm{EI}_{\min }$ and, to a lesser extent, with PoS (Figure 5A-D). This indicates that those laboratory parameters that are important in the evaluation of the patient are also reflected in the oxygen gradient ektacytometry. The number of sickled cells at normoxia and percentage of dense RBCs (DRBCs) both influence Elmax values, as they are significantly correlated (Figure 5E-F), which indicates that Elmax reflects another important factor in the sickling process. These results show how different characteristics such has \% $\mathrm{HbS}, \% \mathrm{HbF}$, sickled cells at normoxia, and \%DRBCs influence different parameters.

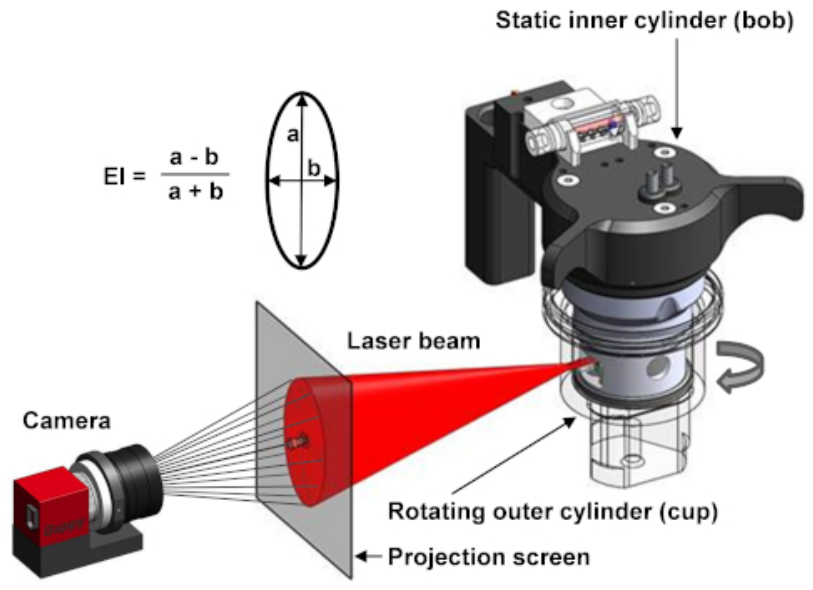

Figure 1. Schematic setup of the ektacytometer. The ektacytometer uses a Couette system to apply shear stress on the cells. A rotation outer cylinder (cup) and a static inner cylinder (bob) are used to induce shear stress by the creation of laminar flow at $37^{\circ} \mathrm{C}$. Between the bob and cup there is a small gap in which the blood suspension is injected. A laser beam shines from the bob through the blood suspension and is scattered by the presence of RBCs. The diffraction pattern is projected and analyzed by a camera. The elongation index (EI) is calculated with the height (a) and the width (b) of the diffraction pattern ${ }^{4}$. Please click here to view a larger version of this figure. 


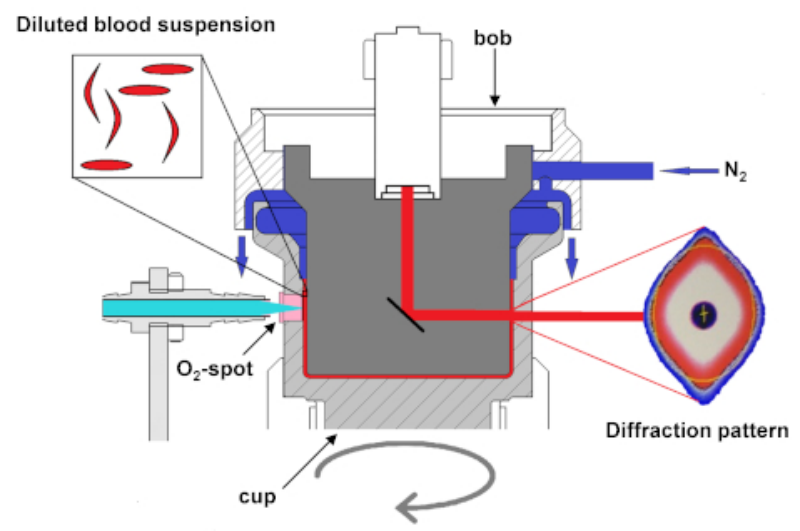

Figure 2. Schematic setup of the ektacytometer with oxygen gradient ektacytometry module. Schematic diagram of the module that shows deoxygenation of the blood suspension slowly with the infusion of nitrogen gas $\left(\mathrm{N}_{2}\right)$. Oxygen tension is measured by the amount of quenching of the luminophore signal sent from the LED-fiber to the $\mathrm{O}_{2}$-spot. Upon deoxygenation, sickle RBCs will start to sickle, their deformability will decrease, and they will no longer align with elliptical RBCs. The sickled RBCs will distort the diffraction pattern, changing its shape from an ellipse to a rhomboid or diamond-like shape. This change in the shape of the diffraction pattern results in a decrease of $\mathrm{EI}_{\text {. }} \mathrm{Measurements}$ of $\mathrm{pO} \mathrm{O}_{2}$ and $\mathrm{El}$ are not performed at the same height in the cup. This ensures better discrimination between the deoxygenation and reoxygenation curves and, hence, a better interpretation of the curve. This figure has been modified from Rab et al. ${ }^{22}$ Please click here to view a larger version of this figure. 
A
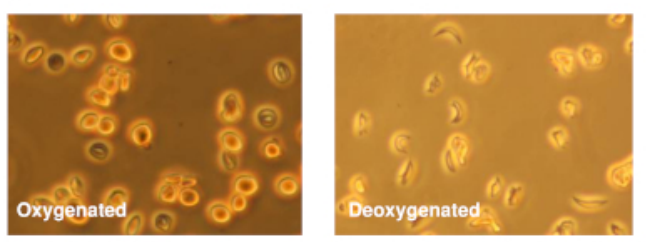

B
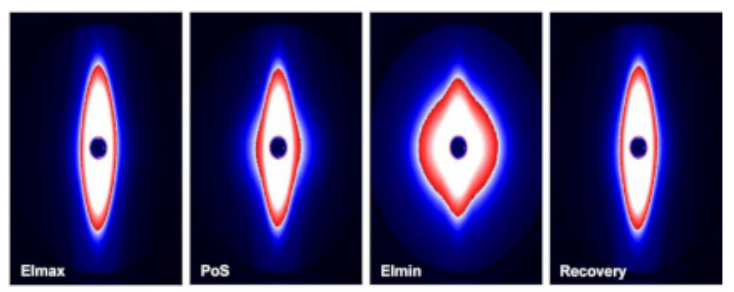

C

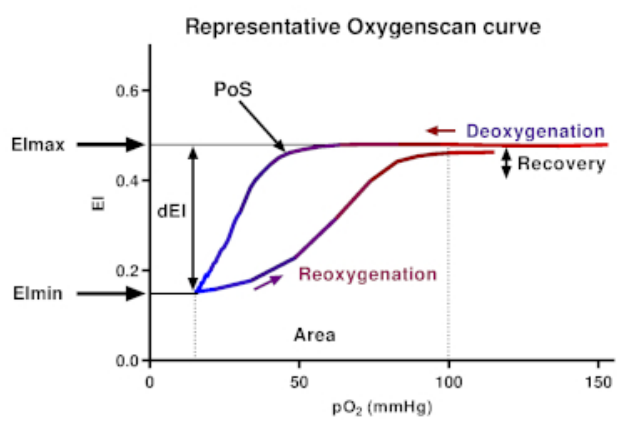

Figure 3. Representative oxygen gradient ektacytometry curve and diffraction patterns. (A) Upon deoxygenation under conditions similar to the oxygen gradient ektacytometry, sickle RBCs were fixed. In control sickle RBCs, the same conditions were used, but without nitrogen gas. Deoxygenated sickle RBCs show a change in shape in contrast to control RBCs. (B) Upon deoxygenation and shear stress (30 Pa), the diffraction pattern changes from an ellipse to a rhomboid. (C) Representative curve of oxygen gradient ektacytometry. The maximum elongation index $\left(\mathrm{EI}_{\max }\right)$ represents the baseline position and shows an overall deformability of the total RBC population. Minimum $\mathrm{El}\left(\mathrm{EI} \mathrm{I}_{\mathrm{min}}\right)$ represents minimal deformability, which is caused by the change in shape and orientation of RBCs upon deoxygenation. $\triangle \mathrm{EI}(\mathrm{dEI}$, the difference in $\mathrm{El}$ between $\mathrm{El}_{\max }$ and $\left.\mathrm{EI}_{\min }\right)$ shows how many cells can sickle during one round of deoxygenation. Point of sickling $\left(\mathrm{PoS}, \mathrm{pO}_{2}\right.$ at $5 \% \mathrm{EI}$ decrease) shows the oxygen tension when the first RBCs start to sickle. The area under the curve (from $\mathrm{pO}_{2 \mathrm{~min}}=100 \mathrm{mmHg}$ ) is calculated in the parameter area. This summarizes $\mathrm{El}_{\max }, \mathrm{El}_{\min }$, and PoS. The capacity of sickled cells to unsickle during reoxygenation is represented in the parameter Recovery (percentage of $\mathrm{EI}_{\max }$ reached during reoxygenation). To aid in the interpretation, all data points were connected in every individual experiment by a line to graphically present the results. This figure has been modified from Rab et al. ${ }^{22}$ Please click here to view a larger version of this figure. 
A

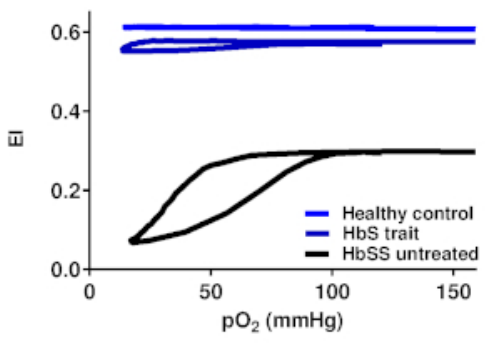

C

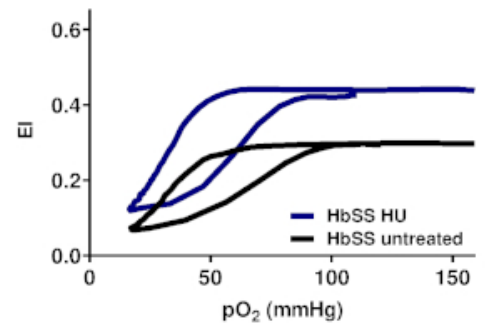

B

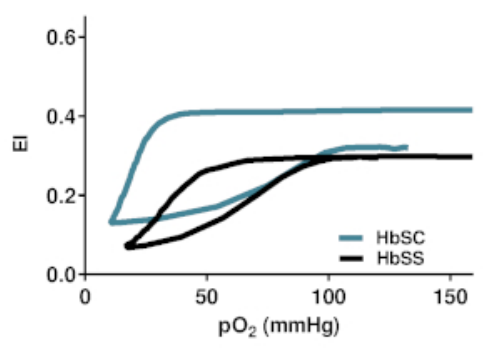

D

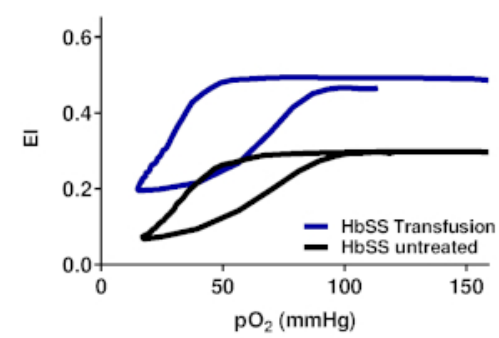

Figure 4. Oxygen gradient ektacytometry parameters correlate with genotype and treatment regimens of SCD patients with SCD. (A) Representative graph of RBCs of HbS carriers (HbS trait) and healthy controls in relation to untreated HbSS patients. (B) Representative graph of RBCs of patients with Hemoglobin SC Disease (HbSC) in relation to untreated HbSS patients. (C) Representative graph of RBCs of hydroxyurea treated homozygous SCD patients (HbSS HU) in relation to untreated HbSS patients. (D) Representative graph of RBCs of HbSS patients treated with blood transfusion (HbSS transfusion) in relation to untreated HbSS patients. This figure has been modified from Rab et al. ${ }^{22}$ Please click here to view a larger version of this figure. 
A

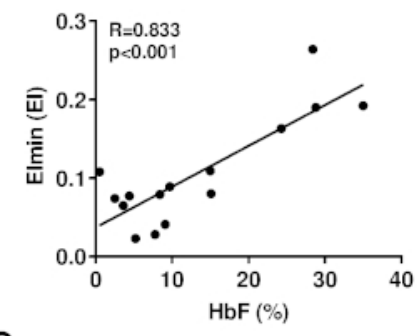

C

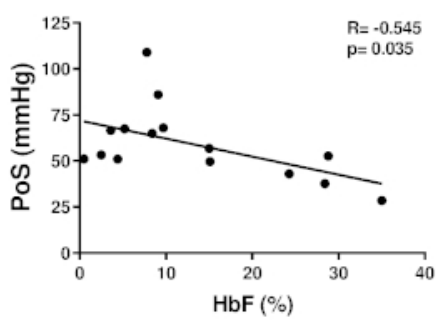

E

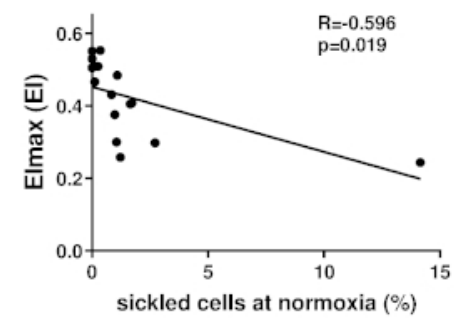

B

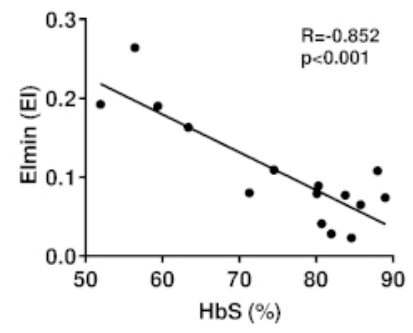

D

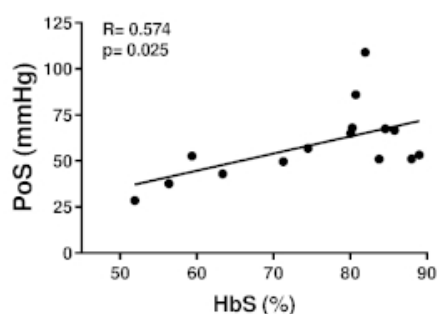

F

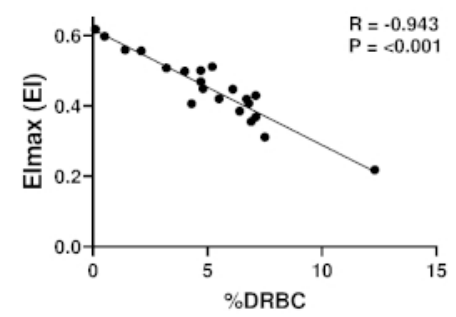

Figure 5. Oxygen gradient ektacytometry parameters are associated with \%HbF, \%HbS, \%sickled cells at normoxia and \%dense RBCs. (A) Linear correlation of minimum elongation index $\left(\mathrm{EI}_{\mathrm{min}}\right)$ and $\% \mathrm{HbF}$ of $15 \mathrm{HbSS}$ or $\mathrm{HbS} / \beta$-thalassemia patients without transfusion. (B) Linear correlation of $\mathrm{El}_{\min }$ and \% $\mathrm{HbS}$. (C) Linear correlation of PoS and \% HbF. (D) Linear correlation of PoS and \% HbS. (E) Linear correlation of maximum $\mathrm{El}\left(\mathrm{EI}_{\max }\right)$ and percent of sickled cells at normoxia measured with digital microscopy. $(\mathbf{F})$ Linear correlation of $\mathrm{EI}_{\max }$ and percentage dense RBCs (\%DRBCs) of 21 patients with HbSS. This figure has been modified from Rab et al. ${ }^{22}$ Please click here to view a larger version of this figure.

\begin{tabular}{|l|l|l|}
\hline Settings & & \\
\hline Files & Storage directory & \\
\hline General options & Default medium viscosity & Viscosity of PVP \\
\hline pO2 scan & Minimum aspiration time (s) & 60 \\
\hline & pO2 scan shear stress (Pa) & 30 \\
\hline & Determine pO2 every (S) & 20 \\
\hline & Moving average size & 2 \\
\hline & PO2 scan step; Edit & 0 -OFF; 60 -ON; $1360-$ OFF; 1640 -OFF \\
\hline & Cal. Area between (mmHg) & 10 and 100 \\
\hline & pO2 control & Off (unchecked) \\
\hline
\end{tabular}

Table 1. The preferred setting of the ektacytometer.

\section{Discussion}

Here we describe oxygen gradient ektacytometry, a method that can be used to study the sickling behavior of red blood cells from SCD patients under a range of oxygen concentrations (Figure 4 and Figure 5). In order to obtain reproducible results, it is important to identify the factors that influence the results. For instance, temperature has a large impact on RBC deformability, mostly due to its effects on the thickness of the viscous solution (PVP). We recommend performing a test measurement at the start of the day to thoroughly heat the machine to $37^{\circ} \mathrm{C}$. This will improve the reproducibility of the results. The osmolarity of the viscous solution should be within a narrow range (282-286 mOsm/kg for PVP), because osmolarity influences hydration status, which in turn affects RBC deformability. The $\mathrm{pH}$ and viscosity of PVP should also be tightly regulated. 
Differences in $\mathrm{pH}$ and temperature can influence curves dramatically ${ }^{22}$. Additionally, remaining water in the cup, bob, and tubes, may cause the lysis of RBCs, thereby resulting in incorrect data, because fewer intact RBCs present in the cup will be measured.

Settings to perform oxygen gradient ektacytometry can be adjusted to address specific investigational questions. Preferred settings are listed in Table 1. A deoxygenation time of 1,300 s was chosen based on observations showing that the extension of deoxygenation did not result in a lower $\mathrm{El}_{\mathrm{min}}$ for most patients. In contrast, shortening of the deoxygenation time would hamper the discriminative power of the oxygen gradient ektacytometry. The reoxygenation time was set to $280 \mathrm{~s}$ due to the rapidly resolving $\mathrm{HbS}$ polymers during reoxygenation, and concomitant restoration of El towards values measured prior to the deoxygenation. Shear stress was set to $30 \mathrm{~Pa}$, which is analogous to the osmotic gradient ektacytometry. Lowering this parameter could hamper the discriminative power. Deoxygenation control can be used if a set of deoxygenation speed is applied to every patient sample. In our preferred settings, this option was switched off because the rate of deoxygenation is patientspecific due to the unique hemoglobin dissociation curve. Hence, switching on the deoxygenation control would eliminate this characteristic from the assay. However, this feature of oxygen gradient ektacytometry is still under investigation.

Several well-known factors influence oxygen gradient ektacytometry parameters, namely $\mathrm{pH}$, temperature, and osmolarity. Ektacytometry, especially PoS, is influenced by 2,3-diphosphoglycerate (2,3-DPG) ${ }^{22}$. Also, there is a clear correlation between $\% \mathrm{HbF}^{\mathrm{and}}$ the $\mathrm{EI}_{\mathrm{min}}$, and to a lesser extent PoS (Figure 5A-D). $\mathrm{EI}_{\max }$ is associated with sickle cells at normoxia, which can explain the observation that shortly after a VOC, $\mathrm{RBC}$ deformability at normoxia $\left(\mathrm{EI}_{\max }\right)$, is higher. The latter is caused by the destruction of the most sickled cells, and hence less deformable RBCs during VOC ${ }^{16}$. As shown in Figure 5F, higher \%dense RBCs (defined as RBCs with a hemoglobin concentration $>1.11 \mathrm{mg} / \mathrm{mL}$ ) correlate strongly with a lower $\mathrm{EI}_{\max }$. This indicates that dense cells are an important factor in RBC deformability at normoxia, similar to previously reported results

Standardization of samples is very important for obtaining reproducible results and for distinguishing between different genotypes and treatments. Correcting for RBC count is important, as the number of RBCs influence the intensity of the diffraction pattern. If lower RBC numbers are present in the gap between the bob and cup, the curve will shift upward and to the left. Additionally, the curve will fluctuate, hampering accurate calculation of the parameters, especially the PoS.

A limitation of this technique is that the El value represents an average of all cells, including different subpopulations. Heterogeneity of RBC populations in SCD patients and its influence on ektacytometry measurement has been intensively studied. This resulted in standardization wherein the size of the diffraction pattern is adjusted to a fixed value instead of corrected for the RBC count ${ }^{23,24}$. Whether or not this way of standardization should also be applied to oxygen gradient ektacytometry measurements is currently under study.

Several techniques to measure RBC deformability under hypoxic conditions were developed based on a deoxygenation step that took place outside the ektacytometer ${ }^{25,26,27}$. Under these conditions, differences in cellular behavior were not observed between patients with $\mathrm{HbS}$ traits and healthy controls under physiological $\mathrm{pH}^{25}$. Oxygen gradient ektacytometry, however, clearly shows a low but evident PoS in individuals with $\mathrm{HbS}$ traits (Figure 4A). To date, in routine clinical practice, the only alternative methods to measure the tendency of an individual patient's RBCs to sickle in vitro include a morphology-based sickling assay: RBCs are incubated under conditions that promote $\mathrm{HbS}$ polymerization, such as low oxygen tension or low $\mathrm{pH}$. A fixative is added after incubation and the percentage of sickled cells is manually or digitally counted using light microscopy. Many preclinical and early phase pharmacologic trials use the sickling assay to generate a secondary outcome variable to be able to predict clinical efficacy in $\mathrm{SCD}^{28,29,30,31,32}$. However, it is time consuming, variability is high and sensitivity is low, the technique is not automated and, therefore, labor intensive. Moreover, morphological changes due to sickling might not correlate well with physiological parameters, such as RBC deformability, because it is a 2-dimensional static assay ${ }^{2}$.

Oxygen gradient ektacytometry provides a functional assay of sickling that is rapid and reproducible. This is an in vitro test that does not consider the endothelial surface. However, it does provide functional aspects of sickling behavior and RBC characteristics, making it a promising technique for sickle cell studies. Future applications of the technique include monitoring treatment efficacy in SCD patients, serving as a biomarker for new treatment strategies, studying sickling behavior, and monitoring chimerism after the stem cell transplantation in SCD.

\section{Disclosures}

The authors declare no competing financial interests.

\section{Acknowledgments}

This work was supported in part by a Eurostars grant estar18105 and by an unrestricted grant provided by RR Mechatronics. The authors thank Sisto Hendriks and Jan de Zoeten for their technical support.

\section{References}

1. Clark, M. R., Mohandas, N., Shohet, S. B. Deformability of oxygenated irreversibly sickled cells. Journal of Clinical Investigation. 65 (1), 189196 (1980).

2. Smith, C., Kuettner, J., Tukey, D., White, J. Variable Deformability of Irreversibly Sickled Erythrocytes. Blood. 58 (1), 71-78 (1981).

3. Clark, M., Mohandas, N., Embury, S., Lubin, B. A simple laboratory alternative to irreversibly sickled (ISC) counts. Blood. 60 (3), $659-663$ (1982).

4. DaCosta, L. et al. Diagnostic tool for red blood cell membrane disorders : Assessment of a new generation ektacytometer. Blood Cells, Molecules, and Diseases. 56 (1), 9-22 (2016).

5. Rabai, M. et al. Deformability analysis of sickle blood using ektacytometry. Biorheology. 51 (2-3), 159-170 (2014).

6. Ballas, S. K., Mohandas, N. Sickle red cell microrheology and sickle blood rheology. Microcirculation. 11 (2), $209-225$ (2004). 
7. Connes, P., Alexy, T., Detterich, J., Romana, M., Hardy-Dessources, M. D., Ballas, S. K. The role of blood rheology in sickle cell disease. Blood Reviews. 30 (2), 111-118 (2015).

8. Hierso, R. et al. Effects of oxidative stress on red blood cell rheology in sickle cell patients. British Journal of Haematology. 166 (4), $601-606$ (2014).

9. Mozar, A. et al. Red blood cell nitric oxide synthase modulates red blood cell deformabilityin sickle cell anemia. Clinical Hemorheology and Microcirculation. 64 (1), 47-53 (2016).

10. Clark, M. R., Mohandas, N., Shohet, S. B. Osmotic Gradient Ektacytometry: Comprehensive Characterization of Red Cell Volume and Surface Maintenance. Blood. 61 (5), 899-911 (1983).

11. Parrow, N. L. et al. Measurements of red cell deformability and hydration reflect $\mathrm{HbF}$ and $\mathrm{HbA2in}$ blood from patients with sickle cell anemia Blood Cells, Molecules, and Diseases. 65, 41-50 (2017).

12. Steinberg, M. H., Chui, D. H. K., Dover, G. J., Sebastiani, P., Alsultan, A. Fetal hemoglobin in sickle cell anemia: A glass half full? Blood. 123 (4), 481-485 (2014).

13. Ballas, S. K., Larner, J., Smith, E. D., Surrey, S., Schwartz, E., Rappaport, E. F. Rheologic predictors of the severity of the painful sickle cell crisis. Blood. 72 (4), 1216-1223 (1988).

14. Lande, W. M. et al. The Incidence of Painful Crisis in Homozygous Sickle Cell Disease: Correlation with Red Cell Deformability. Blood. 72 (6), 2056-2059 (1988).

15. Lemonne, N. et al. Does increased red blood cell deformability raise the risk for osteonecrosis in sickle cell anemia? Blood. 121 (15), 30543057 (2013).

16. Ballas, S. K., Smith, E. D. Red blood cell changes during the evolution of the sickle cell painful crisis. Blood. 79 (8), $2154-2163$ (1992).

17. Telen, M. Cellular adhesion and the endothelium: E-selectin, L-selectin, and pan-selectin inhibitors. Hematology/Oncology Clinics of North America. 28 (2), 341-354 (2014).

18. Papageorgiou, D. P. et al. Simultaneous polymerization and adhesion under hypoxia in sickle cell disease. Proceedings of the National Academy of Sciences. 115 (38), 201807405 (2018).

19. Liu, J., Qiang, Y., Alvarez, O., Du, E. Electrical impedance microflow cytometry with oxygen control for detection of sickle cells. Sensors and Actuators, B: Chemical. 255, 2392-2398 (2018).

20. Du, E., Diez-Silva, M., Kato, G. J., Dao, M., Suresh, S. Kinetics of sickle cell biorheology and implications for painful vasoocclusive crisis. Proceedings of the National Academy of Sciences. 112 (5), 1422-1427 (2015).

21. $\mathrm{Li}, \mathrm{Q}$. et al. Kinetic assay shows that increasing red cell volume could be a treatment for sickle cell disease. Proceedings of the National Academy of Sciences. 114 (5), E689-E696 (2017).

22. Rab, M. A. E. et al. Rapid and reproducible characterization of sickling during automated deoxygenation in sickle cell disease patients. American Journal of Hematology. 94 (February), 575-584 (2019).

23. Renoux, C. et al. Importance of methodological standardization of ektacytometric measures of red blood cell deformability in sickle cell anemia. Clinical Hemorheology and Microcirculation. 62 (2), 173-179 (2016).

24. Parrow, N. L. et al. Measuring Deformability and Red Cell Heterogeneity in Blood by Ektacytometry. Journal of Visualized Experiments. (131), 1-9 (2018).

25. Bessis, M., Feo, C., Jones, E. Quantitation of red cell deformability during progressive deoxygenation and oxygenation in sickling disorders (the use of an automated Ektacytometer). Blood Cells. 8 (1), 17-28 (1982).

26. Sorette, M. P., Lavenant, M. G., Clark, M. R. Ektacytometric measurement of sickle cell deformability as a continuous function of oxygen tension. Blood. 67 (6), 1600-1606 (1987).

27. Huang, Z., Hearne, L., Irby, C. E., King, S. B., Ballas, S. K., Kim-Shapiro, D. B. Kinetics of increased deformability of deoxygenated sickle cells upon oxygenation. Biophysical journal. 85 (4), 2374-2383 (2003).

28. Antoniani, C. et al. Induction of fetal hemoglobin synthesis by CRISPR/Cas9-mediated editing of the human $\beta$-globin locus. Blood. 131 (17), 1960-1973 (2018).

29. Abdulmalik, O. et al. Crystallographic analysis of human hemoglobin elucidates the structural basis of the potent and dual antisickling activity of pyridyl derivatives of vanillin. Acta Crystallographica Section D: Biological Crystallography. 67 (12), 1076 (2011).

30. Oder, E., Safo, M. K., Abdulmalik, O., Kato, G. J., Discovery, D. New Developments in Anti-Sickling Agents: Can Drugs Directly Prevent the Polymerization of Sickle Haemoglobin In Vivo? British Journal of Haematology. 175 (1), 24-30. (2016).

31. Oksenberg, D. et al. GBT440 increases haemoglobin oxygen affinity, reduces sickling and prolongs RBC half-life in a murine model of sickle cell disease. British Journal of Haematology. 175 (1), 141-153 (2016).

32. Xu, G. G. et al. Design, Synthesis, and Biological Evaluation of Ester and Ether Derivatives of Antisickling Agent 5-HMF for the Treatment of Sickle Cell Disease. Molecular Pharmaceutics. 14 (10), 3499-3511 (2017). 